\title{
Foreword
}

\section{Cardiac Anesthesia and Critical \\ Care: New Procedures and \\ Dilemmas Inside and Outside Our Operating Rooms}

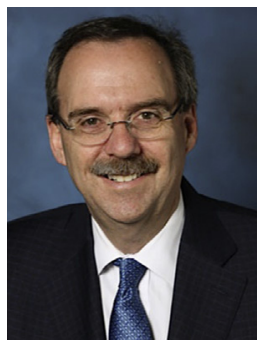

Lee A. Fleisher, MD, FACC, FAHA

Consulting Editor

Cardiac anesthesia as a practice has evolved over the past 50 years. With the advent and increasing use of transesophageal echocardiography, its practitioners acquired a unique skill that required specialty training and eventually certification. Cardiac critical care evolved out of cardiac anesthesia in addition to critical care. Because of the large number of similar procedures with high rates of adverse events, clinical trials could be performed to advance the evidence base for the field. With this long history of focused attention on the field, some would ask about how much advancement would be seen? This issue of Anesthesiology Clinics demonstrates that cardiac anesthesia and critical care are alive and exciting. The editors have invited authors to write articles on advances both inside and outside the operating room, from the question of large-scale clinical trials to the new ethical dilemmas in the field. They created a great source of new information that will educate all of those caring for patients undergoing cardiac surgery, but also those with cardiovascular disease undergoing both surgical and other invasive procedures.

When looking for coeditors for this issue, I turned to 2 rising stars in the field. Karsten Bartels, MD, PhD is Associate Professor of Anesthesiology at the University of Colorado and is Board Certified in anesthesiology, critical care medicine, pain medicine, and advanced perioperative transesophageal echocardiography. He has NIH funding and numerous publications. Stefan Dieleman, $\mathrm{MD}, \mathrm{PhD}$ is Staff Specialist and Clinical Researcher in the Department of Anaesthesia and Perioperative Medicine at the Westmead Hospital in Sydney and Adjunct Senior Lecturer in the Department of Anaesthesia and Perioperative Medicine at the Alfred 
Hospital/Monash University in Melbourne. He focuses his research on clinical trials in cardiac anesthesia. Together, they have created an important monograph for all to learn from.

Lee A. Fleisher, MD, FACC, FAHA Perelman School of Medicine University of Pennsylvania 3400 Spruce Street, Dulles 680

Philadelphia, PA 19104, USA

E-mail address: Lee.Fleisher@uphs.upenn.edu 